\title{
Iterative Receiver Architectures for MIMO-OFDM
}

\author{
Jos Akhtman and Lajos Hanzo \\ School of ECS, Univ. of Southampton, SO17 1BJ, UK. \\ Tel: +44-23-80-593 125, Fax: +44-23-80-593045
}

Email: lh@ecs.soton.ac.uk, http://www-mobile.ecs.soton.ac.uk

\begin{abstract}
In this paper we propose a turbo-detected multiantenna-multi-carrier receiver scheme. Following the philosophy of the turbo processing, our turbo MIMO-OFDM receiver comprises a succession of detection modules, namely the channel estimator, the space-time detector and the decoder, which iteratively exchange soft bit-related information and thus facilitate a substantial improvement of the overall system performance. In this paper we analyse the achievable performance of the iterative system proposed with the aim of documenting the various design trade-offs, such as the achievable error-rate performance, the attainable data-rate as well as the associated computational complexity. Specifically, we report a virtually error-free performance for a rate- $\frac{1}{2}$ turbo-coded 8x8-QPSK-OFDM system, exhibiting an effective throughput of $8 \cdot 2 \cdot \frac{1}{2}=8 \mathrm{bits} / \mathrm{sec} / \mathrm{Hz}$ and having a pilot overhead of only $10 \%$, at SNR of $7.5 \mathrm{~dB}$ and normalized Doppler frequency of 0.003 , which corresponds to a mobile terminal speed of about $65 \mathrm{~km} / \mathrm{h}$.
\end{abstract}

\section{INTRODUCTION}

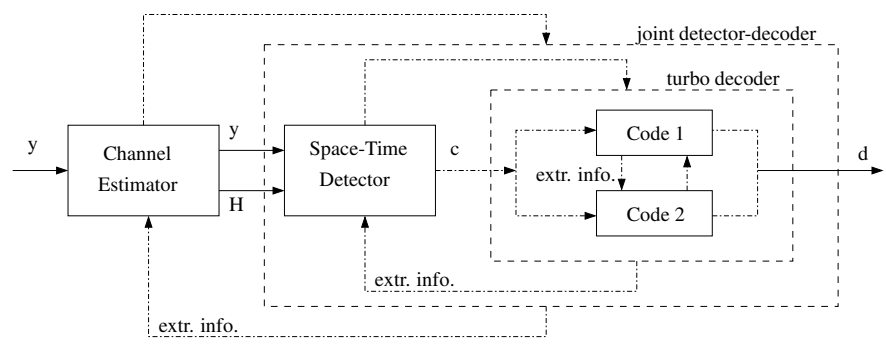

Fig. 1. Schematic of an iterative turbo receiver employing an iterative decision-directed channel estimation, detection and decoding modules.

Despite the immense interest of both the academic and the industrial research communities, the conception of a practical multiple-input multiple-output (MIMO) transceiver architecture, which is capable of approaching the MIMO channel's capacity in realistic channel conditions remains largely an open problem. An important overview encompassing most major aspects of broadband MIMO-OFDM wireless communications including both channel estimation and signal detection, as well as time- and frequency-domain synchronization was contributed by Stüber et al. [1]. Other important publications considering MIMO systems operating in realistic channel conditions include those by Münster and Hanzo [2], Li et. al. [3],

Acknowledgements: The work reported in this paper has formed part of the Wireless Enabling Techniques work area of the Core 4 Research Programme of the Virtual Centre of Excellence in Mobile and Personal Communications, Mobile VCE, www.mobilevce.com, whose funding support, including that of EPSRC, is gratefully acknowledged. Fully detailed technical reports on this research are available to Industrial Members of Mobile VCE.
Mai et. al. as well as Qiao et. al. [4]. Nevertheless, substantial contributions addressing all the major issues pertaining to the design of MIMO transceivers, namely error correction, space-time detection as well as channel estimation in realistic channel conditions remain scarce.

Against this background, in this paper we discuss an iterative, so called turbo multi-antenna-multi-carrier (MAMC) receiver architecture. Our turbo receiver is illustrated in Figure 1. Following the philosophy of turbo processing [5], our turbo MIMO-OFDM receiver comprises a succession of detection modules, which iteratively exchange soft bit-related information and thus facilitate a substantial improvement of the overall system performance.

More specifically, our turbo MIMO-OFDM receiver comprises three major components, namely the soft-feedback aided decision-directed channel estimator (DDCE) [6], followed by the soft-input-soft-output optimized-hierarchy reduced search algorithm (OHRSA) Log-MAP MIMO detector [7] as well as a classic parallel-concatenated soft-input-soft-output turbo code [8]. Consequently, in this paper we would like to analyze the achievable performance of the entire iterative system. Our aim is to document the various design trade-offs, such as the achievable error-rate performance, the attainable data-rate as well as the associated computational complexity.

\section{Channel Estimation For MIMO-OFDM}

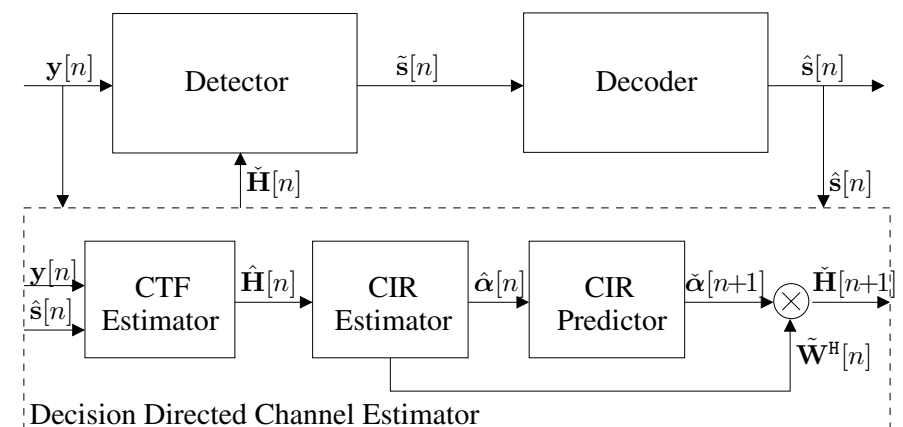

Fig. 2. Schematic of a generic receiver employing DDCE scheme constituted by an a posteriori decision-directed CTF Estimator, followed by a CIR Estimator and an a priori CIR predictor.

The schematic of the channel estimation method considered is depicted in Figure 2. Our channel estimator is constituted by a soft-input a posteriori decision-directed channel transfer function (CTF) estimator [6] followed by a projection approximation subspace tracking (PAST)-aided [9] channel impulse 
response (CIR) etimator [10] and an a priori CIR predictor [10]. As seen in Figure 2, the task of the CTF estimator is to calculate the soft-decision aided temporary estimates $\tilde{\boldsymbol{H}}[n]$ of the CTF coefficients. Subsequently, the task of the CIR estimator is to track the estimates $\hat{\alpha}_{l}[n]$ of the CIR taps, which are then fed into the low-rank time-domain CIR tap predictor of Figure 2 for the sake of producing an a priori estimate $\check{\alpha}_{l}[n+1], l=0,1, \cdots, L$ of the next CIR on a CIR tapby-tap basis [10]. Finally, the predicted CIR is converted to the CTF with the aid of the tranformation matrix $\boldsymbol{W}[n]$ of Figure 2. The resultant CTF is employed by the receiver for the sake of detecting and decoding of the next OFDM symbol.

\section{Iterative ChANNEl Estimation - Detection - DECODING}

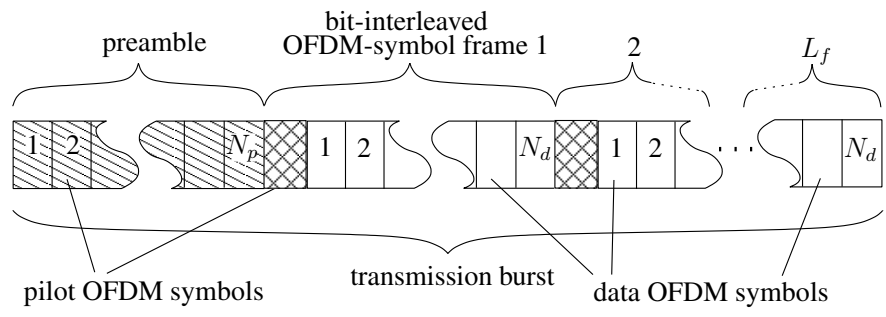

Fig. 3. OFDM transmission burst structure comprising a preamble of $N_{p}$ full-pilot OFDM symbols followed by a sequence of $L_{f}$ data OFDM symbol frames. Each data OFDM-symbol frame is preceded by a single fullpilot OFDM symbol followed by $N_{d}$ information-carying OFDM symbols. Consequently, our OFDM transmission burst accommodates a total number of $N_{p}+L_{f}$ full-pilot OFDM symbols as well as a total number of $L_{f} N_{d}$ information-carrying OFDM symbols.

In this paper we consider the transmission of a sequence of consecutive MIMO-OFDM transmission bursts, which are processed independently. In other words, each of the selfcontained MIMO-OFDM transmission bursts includes all the necessary data, such as for instance pilot signals, required for successful detection and decoding of the information accommodated by the burst. Correspondingly, each MIMOOFDM transmission burst may be processed independently of the neighbouring bursts. This philosophy is reminiscent of the packet-based transmission scheme adopted, for example, in the IEEE $802.11 \mathrm{a} / \mathrm{g}$ WLAN standard [11]. The structure of a single MIMO-OFDM transmission burst considered is depicted in Figure 3. More specifically, our OFDM transmission burst portrayed in Figure 3 commences with a channelsounding preamble formed by $N_{p}$ number of pure pilot MIMO-OFDM symbols. Subsequently, our MIMO-OFDM transmission burst accommodates a sequence of $L_{f}$ number of so-called OFDM-symbol-frames. More explicitely, as seen in Figure 3, each OFDM-symbol-frame constitutes a single bitinterleaved turbo-encoded codeword and comprises a single full-pilot MIMO-OFDM symbol followed by $N_{d}$ number of information-carrying MIMO-OFDM symbols.

For each MIMO-OFDM transmission burst the detection process commences with the initialization of the channel estimator by utilizing the pilot MIMO-OFDM symbols constituting the burst's preamble, as seen in Figure 3. Specifically, both the received signals $\mathbf{y}[n]$ as well as the corresponding transmitted signals $\mathbf{s}[n]$ associated with the $N_{p}$ pilot MIMOOFDM symbols constituting the burst preamble of Figure 3 are sequentially fed into the channel estimator of Figure 2 for the sake of attaining an initial convergence for the three adaptive filters constituting the decision-directed channel estimator of Figure 2.

During the first iteration of the detection process, which is carried out for each subsequent $N_{d}$-OFDM-symbol dataframe of Figure 3 that commences with a full-pilot MIMOOFDM symbol associated with the MIMO-OFDM-symbol index $n$, we perform a long-term prediction of the CIRrelated taps using the CIR tap predictor of Figure 2. More specifically, we aim for predicting the CIR associated with the last OFDM symbol of the current OFDM-symbol-frame of Figure 3, namely the one associated with the MIMOOFDM-symbol index of $\left(n+N_{d}\right)$. The CIRs associated with the remaining $\left(N_{d}-1\right)$ MIMO-OFDM symbols hosted by the current OFDM-symbol-frame are then obtained using linear interpolation between those associated with the $n$th pilot MIMO-OFDM symbol preceding the current OFDMsymbol-frame and the predicted CIR associated with the last $\left(n+N_{d}\right)$ data OFDM symbol. The predicted and interpolated MIMO-CTF coefficients $\check{\mathbf{H}}[m], m=n+1, \ldots, n+N_{d}$ are utilized for the sake of performing an initial detection of the information-carrying data MIMO-OFDM symbols $\mathbf{s}[n]$.

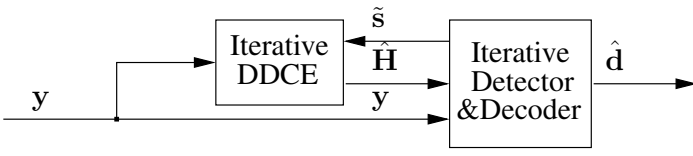

Fig. 4. Schematic of an iterative turbo receiver employing the iterative decision-directed channel estimator of Figure 2 as well as the iterative detection and decoding module.

The resultant tentative estimates of the data-bits $\mathbf{d}$, as well as the associated soft-bit information, corresponding to the entire data MIMO-OFDM-symbol frame of Figure 3 are remodulated in order to generate the soft reference signal $\widetilde{\mathbf{s}}[m], m=n+1, \ldots, n+N_{d}$ [6]. The reference signal $\widetilde{\mathbf{s}}[m]$ is fed back to the soft-input channel estimator [6] for the sake of refining the estimates of the CTF coefficients $\mathbf{H}[m], m=n+1, \ldots, n+N_{d}$. The interaction between the soft-input channel estimator and the iterative MIMO detection and decoding module is illustrated in Figure 4. The iterative channel estimation-detection-decoding process portrayed in Figure 4 is repeated, until a sufficiently reliable detected MIMO-OFDM symbol $\hat{\mathbf{s}}$ is generated.

\section{A. Mitigation of Error Propagation}

The main difficulty associated with the decision-directed approach to channel estimation is constituted by the potential error propagation, where the erroneous data decisions result in erroneous channel estimation, which inflicts further precipitated data decision errors, etc. In other words, the reliability of the estimated CTF coefficients degrades rapidly in the presence of decision errors routinely occurring in the low SNR region. The resultant degradation of the channel state information accuracy results in further decision errors and ultimately in 
divergence of the iterative channel estimation - data detection process and in a subsequent avalanche of decision errors. As was pointed out in [6], the soft feedback aided RLS CTF estimator is capable of substantially mitigating the effects of error propagation. Nevertheless, ensuring the stability of an iterative channel estimation - data detection system in the presence of data decision errors remains a challenging issue. Consequently, for the sake of mitigating the system's vulnerability to error-propagation-related instability effects we propose the following method.

Firstly, after each channel estimation and MIMO detection iteration, which is performed on the $N_{d}$-MIMO-OFDM symbol data frame of Figure 3, we record the resultant MSE. The joint channel estimation and MIMO detection MSE may be expressed as follows

$$
e^{i}[n]=\sum_{m=n+1}^{n+N_{d}} \sum_{k=1}^{K}\left\|\mathbf{y}[m, k]-\hat{\mathbf{H}}^{i}[m, k] \hat{\mathbf{s}}^{i}[m, k]\right\|^{2},
$$

where, as before, $\mathbf{y}[m, k]$ denotes the MIMO signal associated with the $k$ th subcarrier of the $m$ th MIMO-OFDM symbol and recorded at the $n_{\mathrm{r}}$ receive antennas, while $\hat{\mathbf{H}}^{i}[m, k]$ and $\hat{\mathbf{s}}^{i}[m, k]$ are the corresponding estimates of the CTF coefficient matrix and the transmitted signal vector, which were obtained after the $i$ th iteration of the channel estimation and detection process.

Subsequently, after carrying out $i_{\text {ce }}$ number of channel estimation iterations we select the particular pair of CTF estimates $\hat{\mathbf{H}}^{i}[m, k]$ and data estimates $\hat{\mathbf{s}}^{i}[m, k]$, which correspond to the specific iteration resulting in the minimum MSE. More explicitely, the decision rule employed may be expressed as

$$
\{\hat{\mathbf{H}}[m, k], \hat{\mathbf{s}}[m, k]\}=\arg \min _{i} e^{i}[n],
$$

where we have $m=n+1, \cdots, n+N_{d} ; k=1, \cdots, K$ and $i=1, \cdots, i_{\text {ce }}$.

Let us now consider the scenario of encountering a large number of decision errors. Naturally, the decision errors in any of the iterations would result in a degraded channel estimation accuracy in the subsequent iteration and hence even more decision errors as well as an inevitable increase of the corresponding MSE $e^{i}[n]$. Consequently, invoking the final-decision rule of Equation (2) substantially mitigates the system's avalanche-like error propagation and hence improves the system's stability and robustness.

\section{Performance Analysis}

TABLE I

SYSTEM PARAMETERS.

\begin{tabular}{|l|c|}
\hline Parameter & Value \\
\hline \hline Carrier frequency $f_{\mathcal{c}}$ & $2.5 \mathrm{GHz}$ \\
\hline Channel bandwidth $B$ & $8 \mathrm{MHz}$ \\
\hline Number of carriers $K$ & 128 \\
\hline FFT frame duration $T_{\mathcal{S}}$ & $16 \mu \mathrm{s}$ \\
\hline OFDM symbol duration $T$ & $20 \mu \mathrm{s}(4 \mu \mathrm{s}$ of cyclic prefix $)$ \\
\hline Max. delay spread $\tau_{\max }$ & $4 \mu \mathrm{s}$ \\
\hline Max. terminal speed $v$ & $130 \mathrm{~km} / \mathrm{h}$ \\
\hline Norm. Max. Doppler spread $f_{D}$ & $0.006=T \cdot 300 \mathrm{~Hz}$ \\
\hline
\end{tabular}




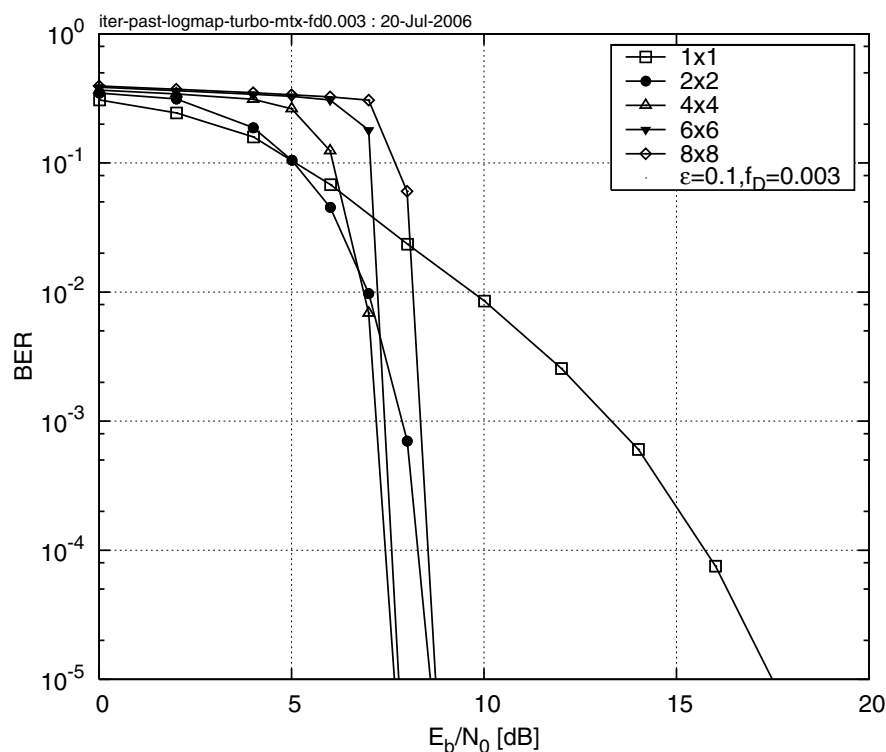

Fig. 6. The BER versus $E_{b} / N_{0}$ performance exhibited by the rate- $\frac{1}{2}$ turbo-coded MIMO-QPSK-OFDM turbo receiver of Figure 4 using $m_{\mathrm{t}} \stackrel{2}{=}$ $n_{\mathrm{r}}=1,2,4,6$ and 8 transmit and receive antennas. The corresponding effective throughputs were $1,2,4,6$ and $8 \cdot 2 \cdot \frac{1}{2}=8 \mathrm{bits} / \mathrm{sec} / \mathrm{Hz}$, respectively. The 7-path COST-207 BU channel model was used [12] and we assumed encountering the Doppler frequency of $f_{D}=0.003$. The pilot overhead of $10 \%$ and the iteration pattern of $\left(i_{\mathrm{ce}}, i_{\mathrm{det}}, i_{\mathrm{dec}}\right)=(3,2,4)$ were used. Additional system parameters are summarized in Table I.

as it does not exceed $m_{\mathrm{t}}=n_{\mathrm{r}}=4$. Furthermore, the BER performance degrades slowly upon further increasing the number of antennas according to $m_{\mathrm{t}}=n_{\mathrm{r}}>4$. The simple explanation of this phenomenon is that as expected, the MIMO-OFDM system benefits from the increased spatial diversity associated with a higher number of antennas. On the other hand, as noted in Section II, the channel estimation problem becomes increasingly more rank-deficient and hence the estimation accuracy of the CIR taps as well as the corresponding subcarrier-related CTF coefficients degrades upon increasing the number of independent spatial links constituting the MIMO channel. The overall system performance is determined by the associated trade-off between the beneficial diversity gain increase and the inevitable degradation of the estimated CTF accuracy. Ultimately, however, the deterioration of the estimated CTF accuracy does not appear to constitute a major impediment. Quantitatively, as evidenced by the results of Figure 6, the BER performance exhibited by the high-complexity system having $m_{\mathrm{t}}=n_{\mathrm{r}}=8$ antennas lies within a $1 \mathrm{~dB}$ margin in comparison to the corresponding BER performance curve associated with the system having $m_{\mathrm{t}}=n_{\mathrm{r}}=4$ transmit and receive antennas. Observe that the $4 \times 4$ system exhibits the best recorded performance and hence appears to represent an optimum tradeoff between the beneficial special diversity gain and the system-size-related channel estimation accuracy degradation.

3) Performance of a Symmetric MIMO System: Subsequently, we would like to characterize the achievable BER performance exhibited by the MIMO-QPSK-OFDM turbo receiver of Figure 4 using various densities of the dedicated pilot MIMO-OFDM symbols. More specifically, in Figure 7

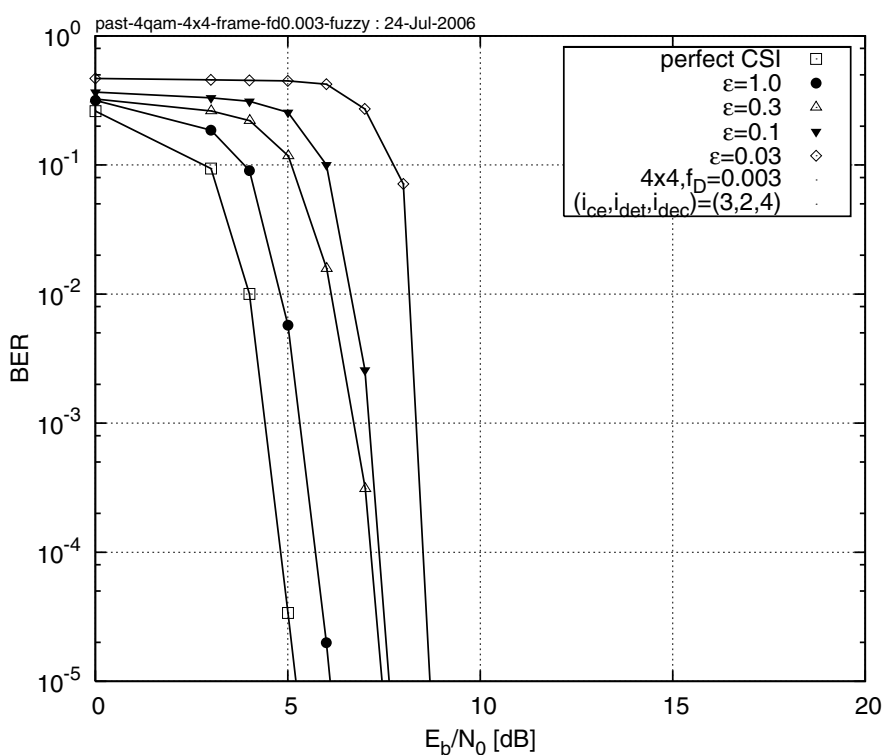

Fig. 7. BER versus $E_{b} / N_{0}$ performance exhibited by the rate- $\frac{1}{2}$ turbo-coded 4x4-MIMO-QPSK-OFDM turbo receiver of Figure 4. The pilot overhead was either 3,10 , 30, or $100 \%$, which corresponds to $\varepsilon=0.03,0.1,0.3$ and 1.0 , respectively, where we consider the idealistic scenario of having $100 \%$ pilots as well as the scenario of perfect channel state information for benchmarking purposes. The 7-path COST-207 BU channel model [12] was used and the Doppler frequency was $f_{D}=0.003$. The iteration pattern of $\left(i_{\mathrm{ce}}, i_{\mathrm{det}}, i_{\mathrm{dec}}\right)=$ $(3,2,4)$ was used and the effective throughput was $4 \cdot 2 \cdot \frac{1}{2}=4 \mathrm{bits} / \mathrm{sec} / \mathrm{Hz}$. All additional system parameters are summarized in Table I.

we have plotted the rate $1 / 2$ turbo-coded QPSK-related BER exhibited by our MIMO-OFDM system employing $m_{\mathrm{t}}=n_{\mathrm{r}}$ transmit and receive antennas. For benchmarking purposes we have included the BER versus $E_{b} / N_{0}$ performance of the MIMO-OFDM system assuming perfect CIR knowledge, as well as assuming channel estimation based on the idealistic scenario of having $100 \%$ pilots. Furthermore, we present our results for the MIMO-OFDM system using pilot overheads of 30,10 and $3 \%$, which corresponds to the pilot overhead ratio of $\varepsilon=0.3,0.1$ and 0.003 , respectively. We observe from Figure 7 that the $100 \%$ pilot-based channel estimation results in an approximately $1 \mathrm{~dB} E_{b} / N_{0}$ degradation in comparison to the perfect CIR estimation scenario. Furthermore, the more realistic assumption of employing up to $10 \%$ dedicated MIMOOFDM pilot symbols results in a further $E_{b} / N_{0}$ degradation of about $1.5 \mathrm{~dB}$ in comparison to the $100 \%$ pilot-based scenario. Additionally, a further reduction of the pilot overhead to as low as $3 \%$ of pilots results in an $E_{b} / N_{0}$ degradation of 2.5 $\mathrm{dB}$ in comparison to the $100 \%$ pilot-based scenario.

4) Performance of a Rank-Deficient MIMO System: Similar phenomena may be observed in Figure 8, which characterizes the achievable BER performance exhibited by a rank-deficient $4 \times 2-M I M O-Q P S K-O F D M$ system. The $4 \times 2$ MIMO scenario constitutes a particularly interesting detection problem. More specifically, let us consider the $k$ th subcarrier of the $n$th MIMO-OFDM symbol. The computational challenge lies in the fact that we have to estimate as many as four transmitted M-QAM symbols $s_{j}[n, k], j=1, \ldots, 4$ as well as the corresponding eight CTF coefficients $H_{i j}[n, k], i=1,2, j=$ $1, \ldots, 4$, while utilising merely the two recorded signal sam- 


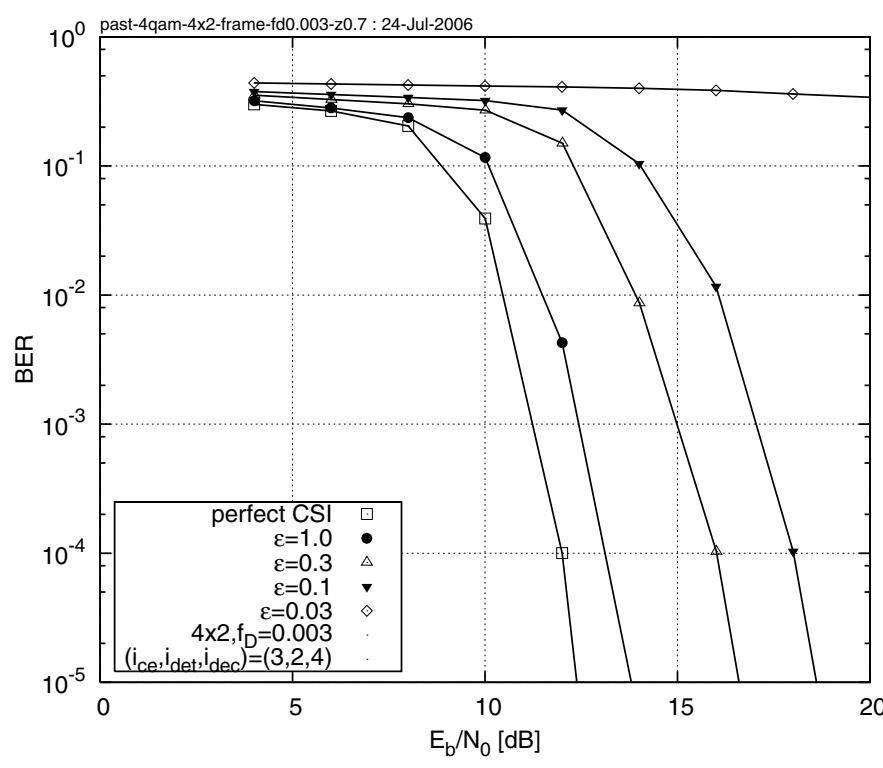

Fig. 8. BER versus $E_{b} / N_{0}$ performance exhibited by the rank-deficient rate- $\frac{1}{2}$ turbo-coded $4 \times 2$-MIMO-QPSK-OFDM turbo receiver of Figure 4 . The pilot overhead was either $3,10,30$, or $100 \%$, which corresponds to $\varepsilon=$ $0.03,0.1,0.3$ and 1.0 , respectively, where we consider the idealistic scenario of having $100 \%$ pilots as well as the scenario of perfect channel state information for benchmarking purposes. The 7-path COST-207 BU channel model was used [12] and the Doppler frequency was $f_{D}=0.003$. The iteration pattern of $\left(i_{\mathrm{ce}}, i_{\mathrm{det}}, i_{\mathrm{dec}}\right)=(3,2,4)$ was used and the effective throughput was $4 \cdot 2 \cdot \frac{1}{2}=$ $4 \mathrm{bits} / \mathrm{sec} / \mathrm{Hz}$. All additional system parameters are summarized in Table I.

ples of $y_{i}[n, k], i=1,2$. Consequently, similarly to Figure 7 we have plotted the BER versus $E_{b} / N_{0}$ performance of the $4 \times 2-M I M O-Q P S K-O F D M$ system assuming perfect CSI as well as assuming channel estimation based on the idealistic scenario of having $100 \%$ pilots. Furthermore, we have plotted the BER corresponding to the scenarios of using pilot overheads of 30,10 and $3 \%$. Similarly to the $4 \times 4$ scenario, assuming $100 \%$ pilot-based channel estimation results in an approximately $1 \mathrm{~dB} E_{b} / N_{0}$ degradation in comparison to the perfect CIR knowledge scenario. On the other hand, in contrast to the $4 \times 4$ scenario characterized in Figure 7, in Figure 8 we may observe that the system employing $10 \%$ of dedicated MIMO-OFDM pilot symbols results in nearly $6 \mathrm{~dB} E_{b} / N_{0}$ degradation in comparison to the $100 \%$ pilot-based scenario. Furthermore, an additional reduction of the pilot overhead to $3 \%$ of pilots results in a system instability and hence no satisfactory BER performance may be achieved, regardless of the SNR encountered.

\section{CONCLUSIONS}

In this paper we have documented the performance trends exhibited by the proposed turbo MIMO-OFDM receiver of Figure 1, which comprises three major components, namely, the soft-feedback decision-directed MIMO channel estimator outlined in Section II, followed by the soft-input-soft-output OHRSA Log-MAP MIMO detector [7] as well as a softinput-soft-output parallel-concatenated turbo code [8]. As seen in Figure 6, we have found that our turbo SDM-OFDM system employing the MIMO-DDCE scheme of Section II as well as the OHRSA Log-MAP SDM detector [7] exhibits a virtually error-free performance for a rate- $\frac{1}{2}$ turbo-coded 8x8-QPSK-OFDM system, having an effective throughput of $8 \mathrm{MHz} \cdot 8 \mathrm{bits} / \mathrm{s} / \mathrm{Hz}=64 \mathrm{Mbps}$ and invoking a pilot overhead of only $10 \%$ at SNR of $7.5 \mathrm{~dB}$ and a normalized Doppler frequency of 0.003 , which corresponds to a mobile terminal speed of about $65 \mathrm{~km} / \mathrm{h}^{1}$.

\section{REFERENCES}

[1] G. Stüber, J. Barry, S. McLaughlin, Y. Li, M. Ingram, and T. Pratt, "Broadband MIMO-OFDM wireless communications," Proceedings of the IEEE, vol. 92, no. 2, pp. 271-294, Feb 2004.

[2] M. Münster and L. Hanzo, "Parallel-interference-cancellation-assisted decision-directed channel estimation for ofdm systems using multiple transmit antennas," Wireless Communications, IEEE Transactions on, vol. 4, no. 5, pp. 2148-2162, Sept. 2005.

[3] Y. Li, J. Winters, and N. Sollenberger, "MIMO-OFDM for wireless communications: signal detection with enhanced channel estimation," IEEE Transactions on Communications, vol. 50, no. 9, pp. 1471-1477, 2002.

[4] X. Qiao, Y. Cai, and Y. Xu, "Joint iterative decision feedback channel estimation for turbo coded v-BLAST MIMO-OFDM systems," in IEEE International Symposium on Communications and Information Technology, vol. 2, 2005, pp. 1384-1388.

[5] L. Hanzo, T. H. Liew, and B. L. Yeap, Turbo Coding, Turbo Equalisation and Space-Time Coding. Chichester, UK; Piscataway, NJ, USA: John Wiley and IEEE Press, 2002, 766 pages. (For detailed contents, please refer to http://www-mobile.ecs.soton.ac.uk.).

[6] R. Otnes and M. Tüchler, "Iterative channel estimation for turbo equalization of time-varying frequency-selective channels," IEEE Transactions on Wireless Communications, vol. 3, no. 6, pp. 1918-1923, 2004.

[7] J. Akhtman and L. Hanzo, "Maximum-Likelihood Advanced Sphere Decoding for MIMO-OFDM," in OFDM and MC-CDMA: A Primer by L. Hanzo and T. Keller. New York, USA: John Wiley, 2006, pp. 253-302.

[8] C. Berrou, A. Glavieux, and P. Thitimajshima, "Near shannon limit errorcorrecting coding and decoding: Turbo codes," in Proceedings of IEEE International Conference on Communications. Geneva, Switzerland: IEEE, May 1993, pp. 1064-1070.

[9] B. Yang, "Projection approximation subspace tracking," IEEE Transactions on Signal Processing, vol. 43, no. 1, pp. 95-107, January 1995.

[10] L. Hanzo, M. Münster, B. J. Choi, and T. Keller, OFDM and MC-CDMA for Broadband Multi-User Communications, WLANs and Broadcasting. John Wiley and IEEE Press, 2003, 992 pages.

[11] Wireless LAN Medium Access Control (MAC) and Physical Layer (PHY) specifications, IEEE 802.11g ed., IEEE LAN/MAN Standards Committee, 2003. [Online]. Available: http://standards.ieee.org/getieee802/802.11.html

[12] M. Failli, "Digital land mobile radio communications COST 207," European Commission, Tech. Rep., 1989.

\footnotetext{
${ }^{1}$ Additional system parameters are characterized in Table I.
} 